\title{
Adaptive Modulation and Coding for Utility Enhancement in VMIMO WSN Using Game Theory
}

\author{
R. Valli and P. Dananjayan
}

\begin{abstract}
The primary component of resource management in wireless sensor network (WSN) is transmitter power control since the sensors are tiny, low cost battery powered devices. The adverse impacts caused by radio irregularities and fading increase the energy consumption and thereby reduce the WSN lifetime. An efficient power control technique is important to sustain system quality and efficiency of wireless sensor networks in such fading conditions. To reduce the fading effects in wireless channel, multi-input multi-output (MIMO) scheme is utilised for sensor network. This paper proposes a power control solution for Virtual MIMO (VMIMO) WSN using game theoretic approach. The game is formulated as a utility maximizing distributed power control game in VMIMO WSN considering the residual energy of the nodes along with adaptive modulation. The proposed algorithm adapts to the changes in channel condition and selects the appropriate modulation and transmits using the optimal transmission power. Simulation results show that the game with pricing provides maximum utility by consuming lesser power.
\end{abstract}

Index Terms-Adaptive modulation and coding, game theory, utility, wireless sensor network.

\section{INTRODUCTION}

Wireless sensor network (WSN) are a network composed of tiny, microelectronic devices capable of sensing, low power computing and wireless communications. Due to the very limited network bandwidth, power and processing capacity, WSN presents remarkable challenges in the fields of wireless networking, communications and signal processing. While a large number of research efforts have been spent on networking and distributed signal processing in order to reduce power consumption, there are few research efforts on energy efficient wireless communications schemes specifically designed for WSN. WSN provides an attractive low-cost technological solution for a wide range of remote sensing and environmental monitoring applications [1]. Due to the large number of wireless sensors in the network and the severe constraints in the system, the wireless communications schemes for WSN should be cost effective and energy efficient.

Transmission power is accountable for up to $70 \%$ of the total energy consumption for off-the-shelf sensor nodes [2]. Therefore energy conservation is very crucial for WSNs, both for each sensor node and the entire network to escalate the network lifetime. In a WSN, each node transmits its information over the air and is prone to fading and other

Manuscript received January 4, 2013; revised April 30, 2013.

The authors are with Adhiparasakthi Engineering College, Melmaruvathur,Tamil Nadu, India (e-mail: valli.r@pec.edu, pdananjayan@ pec.edu). impairments. The data transmitted from the sensor nodes is highly susceptible to error in a wireless environment which leads to higher packet loss and thereby increases the transmit power. Error Control Coding (ECC) is used to improve the system performance and is shown that ECC saves energy as compared to uncoded data transmission [3]. To mitigate the fading effects in wireless channel, diversity techniques can also be used. Multi-Input Multi-Output (MIMO) scheme technology has the potential to enhance channel capacity and reduce transmission energy consumption particularly in fading channels [4]. Another way to combat fading is the use of adaptive modulation which allows a wireless system to choose the highest order modulation depending on the channel conditions while ensuring that no harmful interference is caused to other nodes [5]. Adaptive approaches result in better efficiency by taking advantage of the favourable channel conditions as compared with non-adaptive methods which require a fixed margin to maintain acceptable performance when the channel quality is poor,. After the physical layer set the optimal modulation level, it will adjust the transmission power to stabilize at the implicit optimal transmission power by the feedback based power control scheme. So actually the optimization of modulation level and transmission power is jointly considered.

An approach to adjust the transmission power of a node in WSN is based on game theory which has its roots in economics [6] and there has been a growing interest in applying game theory to study wireless systems [7-9]. Game theory involves a set of competing players looking to maximize their own utility, based upon certain actions they can take. When the users have no knowledge a priori about what others are simultaneously doing, e.g., in a distributed system, the game is non-cooperative, as users have no information on the basis of which they could cooperate. Non-cooperative game theory was first proposed as an efficient framework to formulate the power control problem for wireless data networks without centralized control, e.g., in ad hoc networks [10]. Gao Peng et al., have proposed a non-cooperative power control game for Adaptive Modulation and Coding (AMC) and analysed [11]. But here the energy of the nodes has not been taken into consideration while designing the game.

In this paper, a non-cooperative power control game for adaptive modulation and coding in VMIMO WSN considering the residual energy of the nodes is analysed. The rest of the paper is organized as follows. System model is conferred in Section II. Game theoretic approach for WSN is dealt in Section III. The power control game for adaptive modulation is also formulated in this section. Simulation 
results are given and discussed in Section IV. Finally, conclusions are drawn in Section V.

\section{SYSTEM MODEL}

A two dimensional plane is considered and is assumed to have $N$ nodes in the network area A. All nodes remain stationary after deployment. The energy of the all nodes is limited and own same initial energy except the sink node. The maximum energy of a node is given as $E_{m}$. Energy consumed in the transmission is proportional of bytes transferred and distance. The sensor node has power control capability and is capable of transmitting at variable power levels depending on the distance to the receiver. The node power control range is $\left[s_{\min }, s_{\max }\right]$, where $s_{\max }$ is the maximum transmission power. Nodes are location unaware i.e. they are not equipped with any Global Positioning System (GPS) device.

The source node through local communication broadcasts the data to $\mathrm{M}_{\mathrm{t}}$ active nodes which compose the distributed antenna array. The active nodes are a subset of the total cluster nodes. At this step each active node encodes the transmission sequence according to Space Time Block Coding (STBC) as if each node were a distinct transmit antenna element in the centralized antenna array. This set of $\mathrm{M}_{\mathrm{t}}$ cooperative sensor nodes, communicates with the destination composed of an active set of $M_{\mathrm{r}}$ cooperative sensor nodes. The destination receives data through an $M_{t}$ $x M_{r}$ MIMO channel.

Space time coding schemes are used to improve the performance of MIMO WSN combating the channel fading and interference. The code provides the full diversity over fading channels and improves the quality of signal transmission. Of the space time coding schemes, STBC is more suitable for wireless sensor network with low encoder/decoder complexity. STBC operates on a block of input symbols producing a matrix output whose column represent time and rows represent antennas [12]. They are optimal over all unitary codes with respect to the union bound on error probability. Modulation schemes can be adjusted for the wireless module of the node. In WSNs since depletion of battery resource has a direct impact on the network lifetime, the power control should take into account the residual energy of the nodes. By considering the nodes residual energy, those nodes with minimum residual energy can be used less frequently, thus prolonging lifetime of the node and hence the network.

The Signal to Interference Noise Ratio (SINR) considering the $i^{\text {th }}$ node is given as,

$$
\operatorname{SINR}_{i}=\gamma_{i}=(G) \frac{h_{i} s_{i} \frac{E_{m}}{E_{i}}}{\sum_{j=1, j \neq i}^{N} h_{j} s_{j} \frac{E_{m}}{E_{j}}+N_{o}^{2}}
$$

$G=W / R$ is the processing gain

$W$ is channel bandwidth,

$R$ is the data rate

$E_{i}$ is residual energy of the $i^{\text {th }}$ node

$E_{j}$ is residual energy of the $j^{\text {th }}$ interfering node $s_{i}$ is the transmission power of $i^{\text {th }}$ node $s_{j}$ is the transmission power of $j^{\text {th }}$ interfering node

$E_{m}$ is maximum energy of $i^{\text {th }}$ node

$h$ is the path gain

$N_{O}$ is the noise spectral density

\section{GAME THEORETIC MODELLING}

Nowadays use of game theory in the vast majority of science and applications has increased considerably [13], [14]. The game is defined as a triple $G=\left[N,\left\{S_{i}, M_{i}\right\}, U_{i}\right]$

where $N$ is the set of players, which may be a group of nodes or an individual node in wireless sensor networks. They are the main decision makers of the game.

A set of actions, available for the player $i$ to make a decision. Here each node selects modulation type $\mathrm{m}_{\mathrm{i}} \in \mathrm{M}_{\mathrm{i}}$ and the corresponding $s_{i} \in S_{i}$.

The payoff $\left\{u_{1}, u_{2}, \ldots, u_{i}\right\}$ resulted from the strategy profile. Payoff function expresses the level of income or utility that can be got from the game by the players and is a function of the strategy of all the players.

Each node represents a player, where each player n can adjust its transmit power $\left[s_{\min }-s_{\max }\right]$ to maximize its individual utility function $u_{i}$. However, the wireless environment is inherently interference limited, which results in interactions between nodes. Various utility functions can be considered, but during conditions of mutual interference, these are invariably dependent upon the user SINR. The SINR in turn depends on the transmit powers of all users in the network. Using the standard notation in the game theory literature, we denote the transmit power vector of users other than user $n$ by $s_{-i}$.

The utility function considered for VMIMO WSN is given as 2

$$
u_{i}\left(s_{i}, \gamma_{i}\right)=\eta_{e f f, M C H}=\sum_{i=1}^{M_{R}} M_{\text {sym }} R_{\text {coding }} f\left(\gamma_{i}\right)
$$

where $M_{\text {sym }}$ is the number of bits of each symbol that can be modulated in the $n^{\text {th }}$ type scheme of modulation and coding schemes (MCS), $R_{\text {coding }}$ is the coding efficiency. $f\left(\gamma_{i}\right)$ is the efficiency function which increases with expected SINR.

The efficiency function which is the function of SINR, is given as

$$
f\left(\gamma_{i}\right)=\left(1-2 p_{e}\right)^{F}
$$

where $p_{e}$ is the bit error rate (BER) corresponding to the type of modulation used

$F$ is the size of the packet

Higher order modulation rates are able to offer much high data rates and higher levels of spectral efficiency for the radio communications system, but this comes at a cost. The higher order modulation schemes are considerably less immune to noise and interference. Hence many radio communications systems now use dynamic adaptive modulation techniques. Dynamic modulation technique sense the channel conditions and adapt the modulation scheme to obtain the highest data rate for the prevailing channel conditions. As signal to noise ratio decrease, errors increase thereby increasing the number of retransmission and reduce the throughput. By changing to a 
lower order modulation scheme the link can be made more reliable with fewer data errors and retransmission. The AMC [11] selects appropriate MCS according to the change of channel condition in order to maximize the effective modulation and efficiency. The nodes iteratively decide its transmission power level by maximizing its utility function.

The ideal AMC is given by

$$
\eta_{\text {eff, } A M C}=\max \left(\eta_{\text {eff,MC1 }}, \eta_{\text {eff,MC2 }} \cdots \ldots \eta_{\text {eff,MCn }}\right)
$$

The nodes iteratively decide its transmission power level by maximizing its utility function.

The non cooperative nature of the game means that, an attempt to maximize the utility consumes maximum power, since utility monotonically increases with power. This will also create excessive interference, leading to performance degradation. The solution to this problem is to introduce pricing, which induces a degree of cooperation among players, brings an improvement in system performance by penalizing the selfish nodes and enables the nodes to communicate with a relatively low and stable transmission power.

Pricing accounts for the energy consumed / drained by the sensor node with usage of resources. If the strategy of the $i^{\text {th }}$ node is to transmit at signal power $s_{i} \in S_{i}$ the pricing incurred is a function of $s_{i}$. The class of pricing functions considered is linear and is a monotonically increasing function of transmit power.

The pricing function is given by,

$$
K=z h s_{i} \frac{E_{m}}{E_{i}}
$$

where $z$ is the pricing constant.

The utility with pricing is given by

$$
u_{i}^{c}\left(s_{i}, \gamma_{i}\right)=u_{i}\left(s_{i}, \gamma_{i}\right)-K
$$

\section{Power Control Game based on Energy}

Consider node $i$ is transmitting data to the sink node. Node $i$ broadcasts the data to the cooperative nodes. The cooperative nodes then communicate the data to the destination. The non-cooperative game is an iterative procedure, where at each iteration, the players select the strategy that maximizes their utility function. In other words, a system adapts the most appropriate MCS according to the state of channel condition. This utility function is very important in non-cooperative power control game.

The first step in the game is to determine the threshold SINR. The SINR target requirement is determined based on the modulation type when employing an adaptive modulation scheme. Each node selects modulation type $m_{i} \in M_{i}$ according to the change of SINR in order to maximize the power efficiency and network lifetime [14]. The pseudocode of the procedure explained is given in the algorithm mentioned below.

\section{Algorithm}

$$
\begin{gathered}
\text { Require: } S=\left\{s_{1}, s_{2}, \ldots, s_{\max }\right\} \\
M=\{Q P S K, 16 Q A M, 32 Q A M, 64 Q A M
\end{gathered}
$$

Select the cooperative nodes for communication Determine threshold SINR for $k=1$ to $N_{\text {iter }}$ do

Perform residual energy check

Compute current SINR based on

residual energy using eqn (1).

if current SINR $\geq$ Threshold SINR

Select appropriate AMC end if

Estimate the transmit power from eqn. (2) or (6)

Transmit with optimal power end for

\section{SimUlation RESUlts AND DisCUSSION}

MATLAB acts as a simulation platform in this work. In the area of $100 \mathrm{~m} \times 100 \mathrm{~m}$, random spread of 100 sensor nodes with maximum energy of $4 \mathrm{~J}$ is considered. The simulation parameters considered are given in Table I.

\section{TABLE I: SIMULATION PARAMETERS}

\begin{tabular}{ll}
\hline \hline Simulation Parameters & Description \\
\hline Network area & $100 \times 100 \mathrm{~m}^{2}$ \\
Transmit power $\left\{s_{\min }: s_{\max }\right\}$ & $1: 100 \mathrm{mw}$ \\
Channel Bandwidth & $1 \mathrm{MHz}$ \\
Noise spectral density & $5 \times 10^{-15}$ \\
Path loss component & 2 \\
Modulation techniques & QPSK, \\
& $16-\mathrm{QAM}$, \\
& $32-\mathrm{QAM}$, \\
& $64-\mathrm{QAM}$ \\
Code rates & $1 / 8$, \\
& $1 / 5,1 / 4$, \\
& $1 / 3,1 / 2$, \\
Number of transmit and receive & $2 / 3,4 / 5$ \\
antennas & $2 \times 2, \quad 3 \times 3$, \\
& $4 \times 4$
\end{tabular}

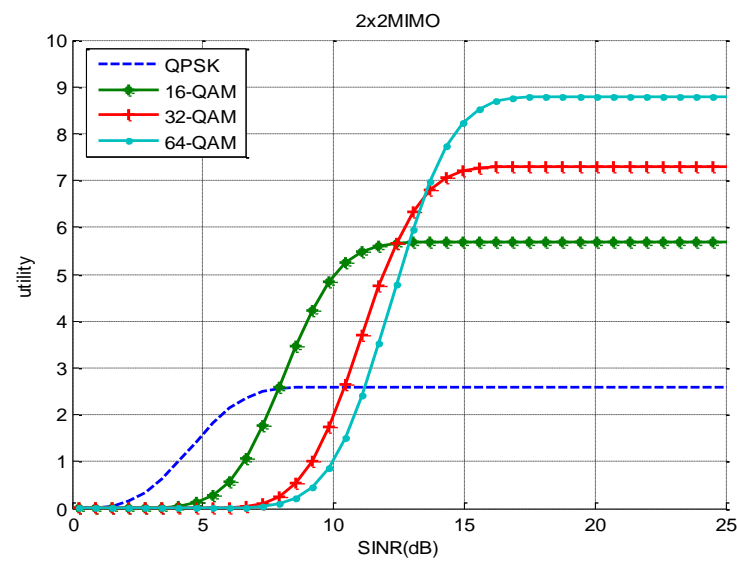

Fig. 1. Utility of the game using 2 transmit and 2 receive antennas for a coding efficiency of $4 / 5$.

The modulation and coding scheme with higher utility needs a higher SINR to operate. Fig. 1 shows the utility of the game with energy check for coding efficiency of 4/5. AMC works by measuring and feeding back the channel SINR to the transmitting node, which then chooses a suitable MCS from the strategy set to maximize the utility. QPSK modulation is adapted during worse channel conditions. It is manifested from figure 1 that, for $2 \times 2$ MIMO configuration the game without pricing provides an utility of $2.7 \mathrm{bits} / \mathrm{s}$, for a SINR of $7 \mathrm{~dB}$ and coding efficiency of $4 / 5$. Higher order modulations 
with higher coding rates are adapted when the channel condition improves. If the current SINR is greater than $8 \mathrm{~dB}$ and less than $12 \mathrm{~dB} 16-\mathrm{QAM}$ is adapted. It is obvious from the figure that at a SINR of $11 \mathrm{~dB}$, the game provides an utility of $5.6 \mathrm{bits} / \mathrm{sec}$ whereas at a SINR of $14 \mathrm{~dB}$, the game without pricing gives an utility of $7.2 \mathrm{bits} / \mathrm{s}$. Considering 64-QAM, it is apparent that at a SINR of $17 \mathrm{~dB}$ and a coding efficiency of $4 / 5$, game without pricing provides a utility of $8.9 \mathrm{bits} / \mathrm{s}$.

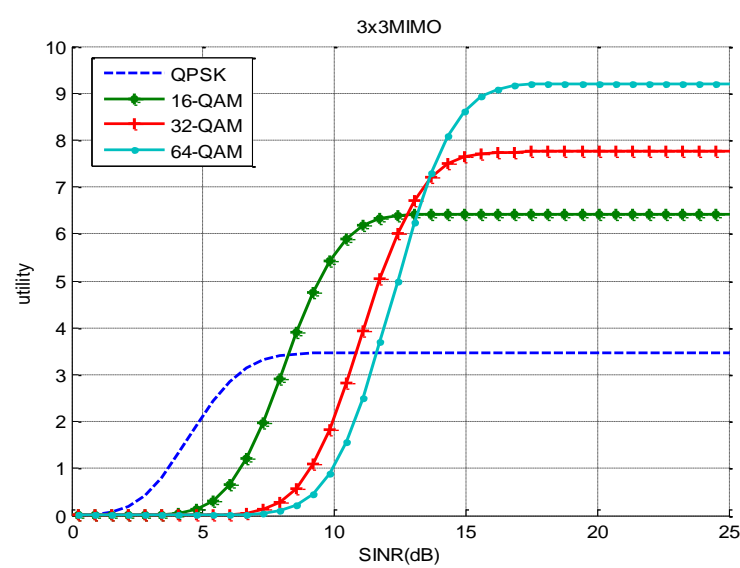

Fig. 2. Utility of the game using 3 transmit and 3 receive antennas for a coding efficiency of $4 / 5$.

From Fig. 2 it is evident that, for $3 \times 3$ MIMO configuration the game without pricing provides an increase in utility by $30 \%$, for a SINR of $7 \mathrm{~dB}$ and coding efficiency of $4 / 5$. It is further observable from the figure that at a SINR of $11 \mathrm{~dB}$, the game provides an utility of $6.4 \mathrm{bits} / \mathrm{sec}$ whereas at a SINR of $14 \mathrm{~dB}$, the game without pricing gives an utility of $7.8 \mathrm{bits} / \mathrm{s}$. Considering 64-QAM, it is apparent that at a SINR of $17 \mathrm{~dB}$ and a coding efficiency of $4 / 5$, game without pricing provides a utility of $9.2 \mathrm{bits} / \mathrm{s}$.

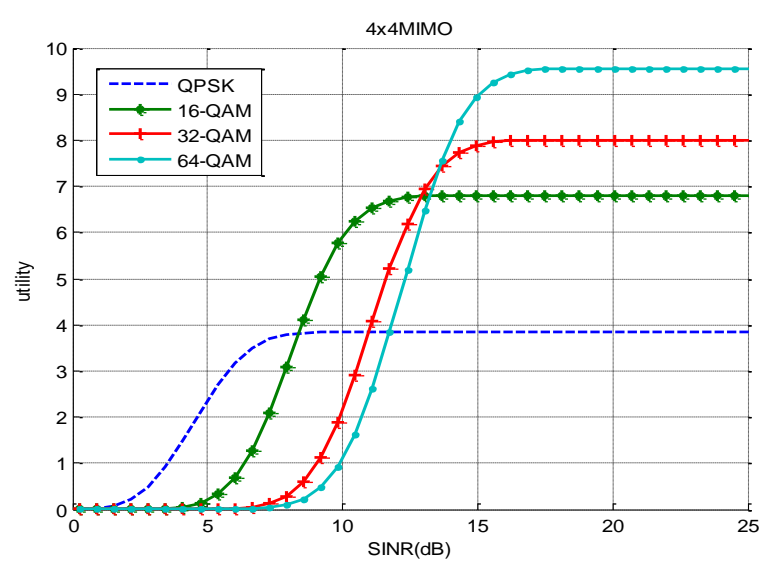

Fig. 3. Utility of the game using 4 transmit and 4 receive antennas for a coding efficiency of $4 / 5$.

Fig. 3 gives the utility of the game taking into account 4 transmit and 4 receive antennas. From this figure it is observed that, for $4 \times 4$ MIMO configuration the game without pricing provides an increase in utility by $12 \%$ compared to $3 \times 3$ MIMO scheme, for a SINR of $7 \mathrm{~dB}$ and coding efficiency of $4 / 5$. An increase in utility by $8 \%$ and $4 \%$ is obtained for 16-QAM and 32-QAM respectively. On adapting 64-QAM, it is noticeable that at a SINR of $17 \mathrm{~dB}$ and a coding efficiency of $4 / 5$, game without pricing provides a utility of $9.6 \mathrm{bits} / \mathrm{s}$.

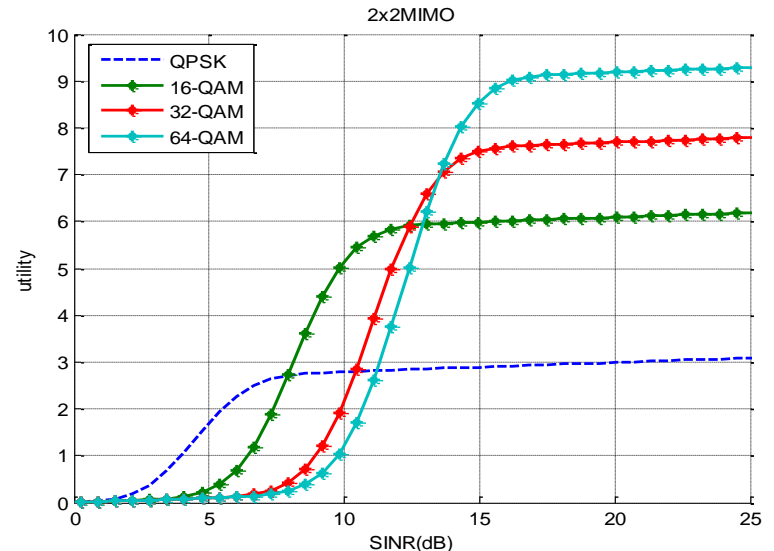

Fig. 4. Utility of the game with pricing using 2 transmit and 2 receive antennas for a coding efficiency of $4 / 5$.

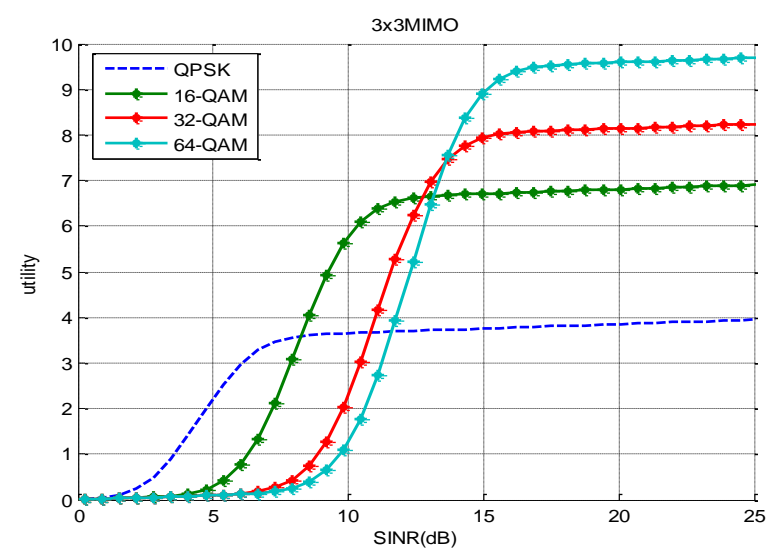

Fig. 5. Utility of the game with pricing using 3 transmit and 3 receive antennas for a coding efficiency of $4 / 5$

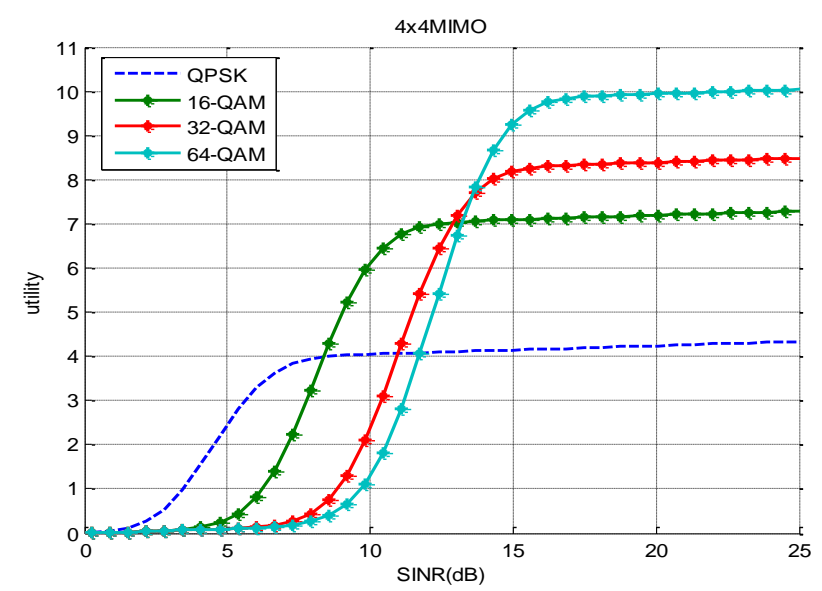

Fig. 6. Utility of the game with pricing using 4 transmit and 4 receive antennas for a coding efficiency of $4 / 5$

Figs. 4-6 give the utility of the game with pricing for $2 \times 2$, $3 \times 3$ and $4 \times 4$ MIMO configuration. Considering the $2 \times 2$ MIMO scheme the game with pricing provides an utility $2.8 \mathrm{bits} / \mathrm{sec}$ for a SINR of $7 \mathrm{~dB}$ thereby providing $4 \%$ increase in utility. An increase in utility by $5.35 \%$ and $3 \%$ is obtained for the 16-QAM and 32-QAM considering the game with pricing. 64-QAM provides a utility of $9.2 \mathrm{bits} / \mathrm{sec}$. On comparing $3 \times 3$ and $4 \times 4$ MIMO schemes with pricing an increase in utility by around $5 \%$ is achieved. 


\section{CONCLUSION}

An energy efficient adaptive modulation and coding for power control in VMIMO-WSN using game theoretic approach taking into account the residual energy of the nodes has been analysed. The game is designed such that, appropriate modulation and coding is selected based on the current channel condition. The utility of nodes residual energy check for $2 \times 2,3 \times 3$ and $4 \times 4$ MIMO schemes are compared. The maximum utility is obtained as the order of diversity increases. With the inclusion of pricing the interference among the nodes due to the optimizing behaviour of a particular node is suppressed. Further the outcome shows that employing higher order MIMO configuration with pricing achieves the best response for the sensor nodes.

\section{REFERENCES}

[1] F. Akyildiz, W. Su, Y. Sankarasubramaniam, and E. Cayirci, "A survey on sensor networks," IEEE Communications Magazine, vol. 40, no. 8, pp.102-114, August 2002.

[2] D. Lymberopoulos, A. Lindsey, and Savvides, "Characterization of the radio signal strengh variability in 3-D IEEE 802.15.4 networks using monopole antennas," in Proc. Third European Workshop on Wireless Sensor Network, Zurich, 2006, pp. 326-341.

[3] S. Chouhan, R. Bose, and M. Balakrishnan, "A framework for energy consumption based design space exploration for wireless sensor nodes," IEEE Transaction on Computer-Aided Design of Integrated Circuits and Systems, vol. 28, no. 7, pp. 1017-1024, July 2009.

[4] S. Cui, Andrea. J. Goldsmith, and A. Bahai, "Energy efficiency of MIMO and cooperative MIMO techniques in sensor networks," IEEE Journal on Selected Areas in Communications, vol. 22, no. 6 , pp.1089-1098, August 2004.

[5] A. J. Goldsmith and S.-G. Chua, "Adaptive coded modulation for fading channels," IEEE Transactions on Communications, vol. 46, no. 5, pp. 595-602, May 1998.

[6] D. Fudenberg and J. Tirole, Game theory, MIT Press, Cambridge, MA, 1991.

[7] C. U. Saraydar, N. B. Mandayam, and D. J. Goodman, "Efficient power control via pricing in wireless data networks," IEEE Transactions on Communication, vol. 50, no. 2, pp. 291-303, August 2002.

[8] M. Hayajneh and C. T. Abdallah, "Distributed joint rate and power control game-theoretic algorithms for wireless data," IEEE Communication Letter, vol. 8, no. 8, pp. 511-513, August 2004.

[9] A. B. MacKenzie and S. B. Wicker, "Game theory in communications: Motivation, explanation, and application to power control," in Proc. IEEE Global Telecommunications Conference, San Antonio, TX, USA, November 2001, pp. 821-826.
[10] D. Goodman and N. Mandayam, "Power control for wireless data," IEEE Personal Communication Magazine, vol. 7, no. 2, pp. 48-54, Apr. 2000

[11] P. Gao, D.-X. Meng, N. Cheng, S.-C. Liang, and G.-F. Tu, "Non-cooperative power control game for adaptive modulation and coding," The Journal of China Universities of Posts and Telecommunications, vol. 17, no. 3, pp. 31-37, June 2010.

[12] V. Tarokh, H. Jafarkhani, and A. R. Calderbank, "Space-time block codes from orthogonal designs," IEEE Transactions on Information Theory, vol. 45, no. 5, pp. 1456-1467, 1999.

[13] S. Sengupta, M. Chatterjee, and K. A. Kwait, "A Game theoretic framework for power control in wireless sensor networks," IEEE Transactions on Computers, vol. 59, no. 2, pp. 231-242, February 2010.

[14] R.Valli and P. Dananjayan, "Energy efficient adaptive modulation for power control in WSN using game theoretic approach," in Proc. International Conference on Information and Communication Technolgy, Chennai, 24 ${ }^{\text {th }}$ Decemebr 2011, pp. 58-64.

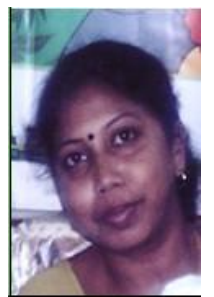

R. Valli received B.E degree in electronics and communication engineering from Madras University, Chennai in 1996 and M. Tech degree in 2005 and Ph.D. in 2012 from Pondicherry University. She has fifteen years' teaching experience. She is currently working as an associate professor in the Department of Information Technology at Adhiparasakthi Engineering College,Melmaruvathur, India. She has published several papers in International Journals and International Conferences. She has visited Malaysia to present a paper in an International Conference. Her research interests include computer networks, wireless ad hoc and sensor networks.

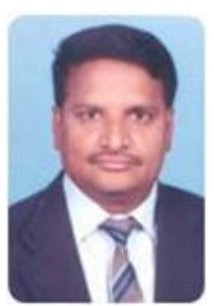

P. Dananjayan received Bachelor of science from University of Madras in 1979, Bachelor of Technology in 1982 and Master of Engineering in 1984 from the Madras Institute of Technology, Chennai and Ph.D. degree from Anna University, Chennai in 1998. He is working as professor in the Department of Electronics and Communication Engineering, Pondicherry Engineering College, Pondicherry, India. He has been as visiting professor to AIT, Bangkok. He has more than 120 publications in National and International Journals. He has presented more than 160 papers in National and International Conferences. He has guided $12 \mathrm{Ph} . \mathrm{D}$. candidates and is currently guiding 7 Ph.D. students. His research interests include spread spectrum techniques, wireless communication, wireless adhoc and sensor networks. 